(pp 159-166)

\title{
The Effect of Combined Functional Training with Muscle Strengthening on Everyday Function in Community-dwelling Elderly Individuals
}

\author{
Hiroyuki HASHIDATE, PT, PhD, Taizo SHIOMI, PT, PhD \\ Department of Physical Therapy, School of Health Sciences, Kyorin University \\ Hiroyuki SHIMADA, PT, PhD \\ Center for Development of Advanced Medicine for Dementia, National Center for Geriatric and Geron- \\ tology \\ Norio SASAMOTO, MD, PhD \\ Yamanashi Care Academy
}

Purpose: The purpose of this study was to assess the difference of between the effect of muscle strengthening and combined functional training with muscle strengthening on everyday function in community-dwelling elderly individuals who had risks for functional decline.

Subjects: Sixty eight specified elderly subjects using adult day-care service were participated in the study, and were assigned to muscle strength group and functional training group.

Methods: The muscle strength group $(n=40)$ performed resistance training with leg press machine which intensity was $60 \%$ maximum voluntary contraction, and conducted strength training with weight in lower extremity muscles. The functional training group $(n=28)$ were executed balance and gait exercise consisted of standing balance exercise on form mat and tandem walking, and walking exercise with weight and long distance walking, in addition to muscle strength training. The subjects were measured 1 repetition maximum of leg press machine (1RM), one leg standing time (OLS), functional reach test (FR), timed "Up \& Go" test (TUG), maximum walking speed (MWS), Tokyo Metropolitan Institute of Gerontology index, the basic checklist and self-perceived health in before and after the training. The exercise intervention was performed for 3 months, 1 time weekly.

Results: The functional training group showed significant improvements in TUG, MWS and selfperceived health than the muscle strength group after exercise intervention. There were main effects in 1RM, OLS, FR, however, no interactions were found.

Conclusion: The results suggested that the combination of functional training and muscle strength training was useful to maintain/improve gait performances and self-perceived health than muscle strength training only in the specified elderly individuals. 